\title{
Evidence of increased radio-frequency losses in cavities from the fundamental power coupler cold window
}

\author{
Frank Marhauser@ and Gianluigi Ciovati๑* \\ Thomas Jefferson National Accelerator Facility, Newport News, Virginia 23606, USA
}

(Received 23 July 2021; accepted 7 September 2021; published 27 September 2021)

\begin{abstract}
High radio-frequency (rf) losses measured for cavities in original Continuous Electron Beam Accelerator Facility (CEBAF) cryomodules, compared to the losses measured in single-cavity tests, have been a longstanding issue related to their performance. We summarize experimental evidence of increased of losses in CEBAF cavities arising from the fundamental power coupler cold window and waveguide, respectively. Cryogenic rf tests were done on cavities tested in vertical cryostats as well as inside cryomodules in the accelerator tunnel. The cold window metallization losses were assessed by combining numerical results with measured data obtained with an existing cryogenic waveguide resonator setup. The results showed that the cold window metallization losses can increase the cavity of heat load at $2.07 \mathrm{~K}$ by up to $86 \%$, depending on the standing-wave pattern in the fundamental power coupler waveguide, and that such losses are reduced if the distance between the waveguide and the cavity cells is increased.
\end{abstract}

DOI: 10.1103/PhysRevAccelBeams.24.092001

\section{INTRODUCTION}

The Continuous Electron Beam Accelerator Facility (CEBAF) is a continuous-wave electron accelerator which has been operating at Jefferson Lab since 1994 [1,2]. Acceleration of the electron beam is provided by 418 $1497-\mathrm{MHz}$ superconducting radio-frequency (SRF) cavities cooled to a temperature of $2.07 \mathrm{~K}$. Four different types of cryomodules (CMs), identified by the label Cxxx, where $x x x$ is the design energy gain, are currently operating in CEBAF: (a) C20 cryomodules, containing eight five-cell cavities which are part of the original installation of CEBAF in the 1990s; (b) C50 cryomodules, containing eight five-cell cavities which were fabricated as part of the original CEBAF installation but were reprocessed with upto-date treatment procedures, in the years 2007-2017 [3]; (c) $\mathrm{C} 100$ cryomodules, containing eight seven-cell cavities with a new cell shape, installed in 2011-2013 [4]; and (d) C75 cryomodules, containing eight five-cell cavities with end groups recycled from original CEBAF cavities but with a new cell shape [5], the first such cryomodule installed in CEBAF in June 2021.

The results from the commissioning of the $\mathrm{C} 20 \mathrm{CMs}$ in the CEBAF tunnel in the 1990s showed that the cavity rf heat load was about a factor of 2 higher than measured in

\footnotetext{
"gciovati@jlab.org
}

Published by the American Physical Society under the terms of the Creative Commons Attribution 4.0 International license. Further distribution of this work must maintain attribution to the author(s) and the published article's title, journal citation, and DOI. vertical test cryostats during the cavity qualification process, even at low accelerating gradient, without field emission [2]. This issue persisted during the C50 refurbishment program [3]. Significant efforts were made in recent years to understand the contribution of the remanent magnetic field from either magnetized components or inadequate magnetic shielding close to the cavities [6], and these have been largely addressed in the last C50 cryomodule (1L13), installed in CEBAF in 2017. Nevertheless, the $Q_{0}$ of the C50 cavities in this cryomodule was about $30 \%$ lower than measured in a vertical test cryostat at Jefferson Lab's vertical test area (VTA) [7]. Figure 1 shows representative curves of the quality factor $Q_{0}$, measured as a function of the accelerating gradient $E_{\text {acc }}$, at $2.07 \mathrm{~K}$ for $\mathrm{C} 20, \mathrm{C} 50$, and $\mathrm{C} 75$ cavities tested in the VTA and in a cryomodule.

All CMs in CEBAF employ two rf ceramic windows located close to the cavities, within or external to the CMs, as part of the fundamental power coupler (FPC) waveguide. The space between two rf windows is actively pumped. This provides a guard vacuum between the ultrahigh vacuum (UHV) in the cavity and beam line space and the atmospheric pressure as a safeguard to protect the UHV from direct air-to-vacuum leaks in case a single window would break and leak (e.g., due to prolonged arcing or $\mathrm{rf}$ breakdown). The $\mathrm{C} 100$-style CMs have been designed to accommodate two warm rf windows along the FPC positioned at some distance away from the cryogenic environment [8]. Unlike the $\mathrm{C} 100$ cavities being each embedded in an individual helium vessel, C20, C50, and C75 cavities share a common helium vessel as cavity pairs, as shown schematically in Fig. 2 [9]. The rf window nearest 


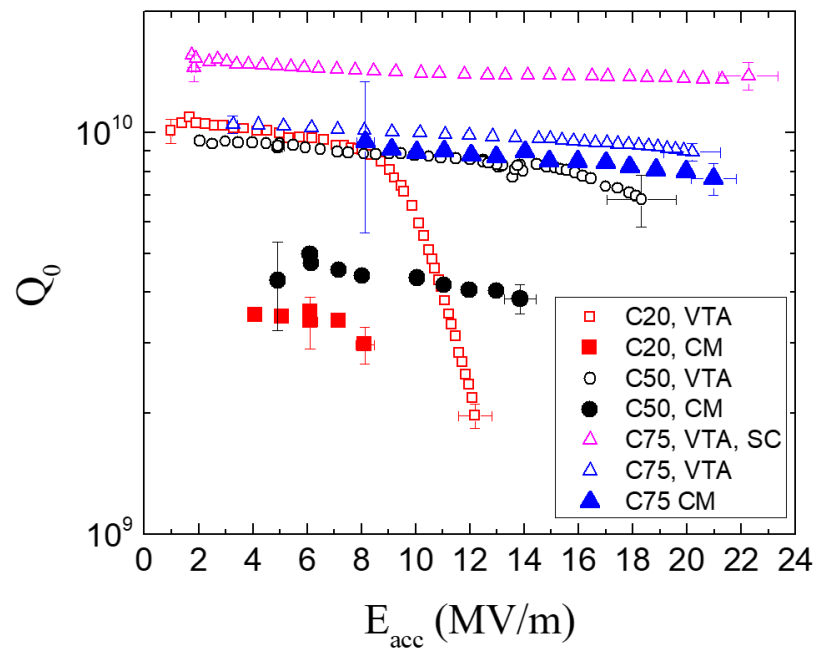

FIG. 1. $Q_{0}\left(E_{\text {acc }}\right)$ measured at $2.07 \mathrm{~K}$ for typical C20, C50, and C75 cavities. The C20 cavity serial number (SN) IA137 was tested in the VTA as part of a cavity pair in June 1992 and in CM 1 L17 during its recommissioning in July 2013. The C50 cavity SN IA075 was tested in the VTA as part of a cavity pair in April 2009 and in CM 1L04 during its recommissioning in April 2013. The C75 cavity SN 5C75-RI-006 was tested in the VTA as a single cavity (SC) in Nov. 2019, then as part of a cavity pair in February 2020, and finally in CM C75-01 in April 2021.

to the cavity is located within a $\mathrm{Nb}$ flange, with all but one side immersed in the liquid helium (LHe) bath. Henceforth, we will refer to this window as the cold window.

As part of the C50 and C75 refurbishment programs, every FPC waveguide is equipped with a waveguide chicane (a so-called dogleg) made from niobium (see Fig. 3, right). The dogleg is keeping the ceramic out of sight for the electron beam. The intent is to eliminate charge buildup at the ceramic surface, which will ultimately result in arcing across or through the cold window [10]. A dogleg is installed between a cavity and the helium vessel in place of the formerly straight ( 14-cm-long) $\mathrm{Nb}$ waveguide extension. Thereby, the cold window is shifted further

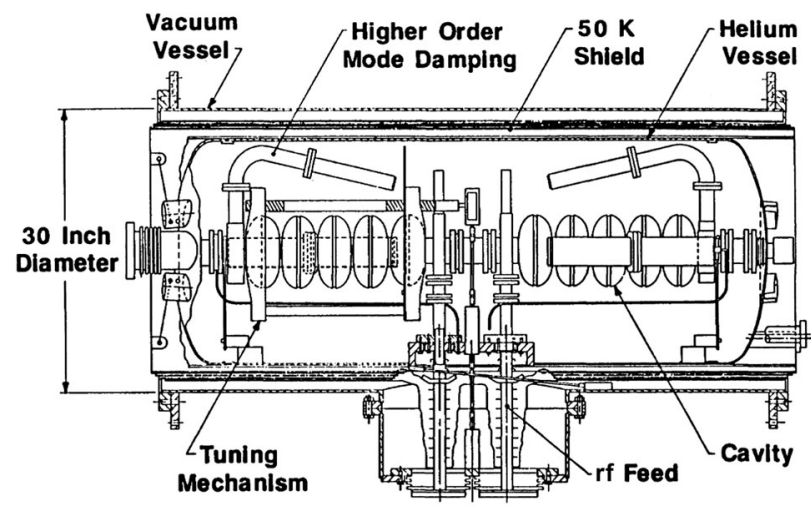

FIG. 2. Schematic of one-quarter section of an original CEBAF cryomodule, with a cavity pair inside the $\mathrm{He}$ vessel (from Ref. [9]).
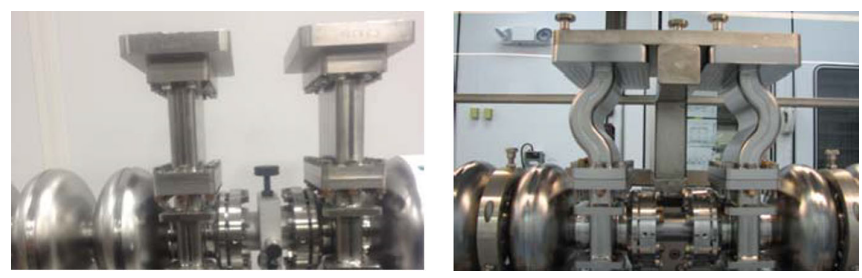

FIG. 3. Close-up picture of the FPC waveguide section for original C20 cavities (left) and after the refurbishment using doglegs (right) as installed in C50 and C75 cryomodules.

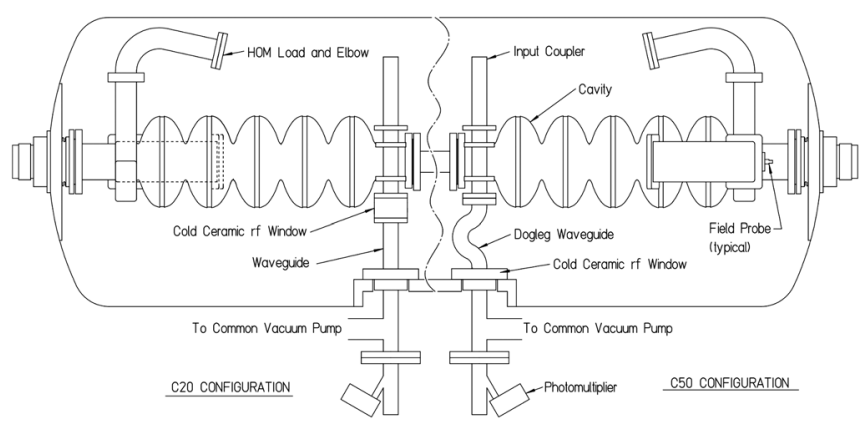

FIG. 4. Schematic layout of a cryounit showing the cold and warm rf ceramic window locations within a $\mathrm{CM}$ for both a C20 CM configuration and a C50 or C75 CM configuration.

away from the cavity. After refurbishment, it is located within the outer dogleg flange, which itself ends at the cavity-pair helium vessel interface. These details are illustrated in Fig. 4, showing a schematic layout of a C20 and C50 cavity inside the CM to highlight the location of the cold windows in each configuration. The C75 cavities have the same CM configuration as the C50 cavities.

The nominal operational parameters are summarized in Table I for C20, C50, and C75 cavities. It includes the required minimal forward power $P_{b}$ to achieve the nominal accelerating gradient $E_{\text {acc }}$ in each of the different cavities at the maximum beam loading of $460 \mu \mathrm{A} ; P_{c}$ denotes the cavity SRF wall losses equivalent to the specified unloaded quality factor $Q_{0}$, typically measured at $2.07 \mathrm{~K}$, which is the nominal CEBAF cavity operating temperature.

To examine possible $Q_{0}$ degradation mechanisms due to the cold window in various test configurations, we have analyzed heat load and quality factor measurements for

TABLE I. Nominal cavity performance parameters of cavities installed in original CEBAF cryomodules.

\begin{tabular}{lccccc}
\hline \hline $\begin{array}{l}\text { Cavity } \\
\text { type }\end{array}$ & $\begin{array}{c}E_{\text {acc }} \\
(\mathrm{MV} / \mathrm{m})\end{array}$ & $Q_{0}(2.07 \mathrm{~K})\left(\times 10^{9}\right)$ & $\begin{array}{c}P_{c} \\
(\mathrm{~W})\end{array}$ & $\begin{array}{c}P_{b} \\
(\mathrm{~kW})\end{array}$ & $\begin{array}{c}Q_{\text {ext FPC }} \\
\left(\times 10^{6}\right)\end{array}$ \\
\hline C20 & 5 & 2.4 & 5.4 & 1.2 & $6.6 \pm 20 \%$ \\
C50 & 12.5 & 6.8 & 11.9 & 2.9 & $8.0 \pm 25 \%$ \\
C75 & 19.1 & 8.0 & 20.9 & 4.3 & $20 \pm 15 \%$ \\
\hline \hline
\end{tabular}


cavities in the following setups: (i) C75 cavities tested as individual cavities and as cavity pairs in vertical dewars; (ii) C50 cavity IA366 tested individually in a vertical dewar and in a cavity-pair-like configuration; (iii) FEL04, a C20 cryomodule installed in JLab's free-electron laser (FEL); (iv) $1 \mathrm{~L} 13$, a refurbished C50 cryomodule installed in CEBAF's north linac. 1L13 also houses the first two C75 prototype cavities installed in CEBAF [7].

Before analyzing the main experimental results in Sec. III, we first provide some insight in the possible cold window loss mechanism in Sec. II. It includes an assessment of the cold window metallization losses utilizing measurement data carried out in a dedicated cryogenic cold window waveguide resonator. The results yields a realistic assessment of the electrical conductivity and, thus, the normal conducting metallization losses for usage in numerical simulations. This is of high importance for all subsequent numerical simulations quantifying the $Q_{0}$ degradation of cavities tested in a vertical cryostat and eventually in cryomodules, as addressed in Sec. IV. The experimental data are accompanied by numerical results as far as possible to explain some of the involved rf loss mechanisms. For instance, the impact of using waveguide stub tuners on the $Q_{0}$ degradation is addressed in Sec. IV B. Eventually, an estimate is provided for the $Q_{0}$ degradation due to the rf heat load conducted into the liquid helium bath arising in the copper-plated waveguide beyond the cold window location.

\section{ORIGIN OF COLD WINDOW LOSSES}

To better understand the rf loss mechanisms that can arise in a cold window, several numerical simulations have been carried out already in past years [11]. The actual details of the cold window design are important to incorporate in a 3D rf model as much as feasible and are provided in Fig. 5.

Cold windows are made from high-purity, polycrystalline alumina $\left(\mathrm{Al}_{2} \mathrm{O}_{3}\right)$ with a relative permittivity $\epsilon_{r}$ of $\sim 9$ at $1.5 \mathrm{GHz}$. The loss tangent $\tan \delta$ of the ceramic body

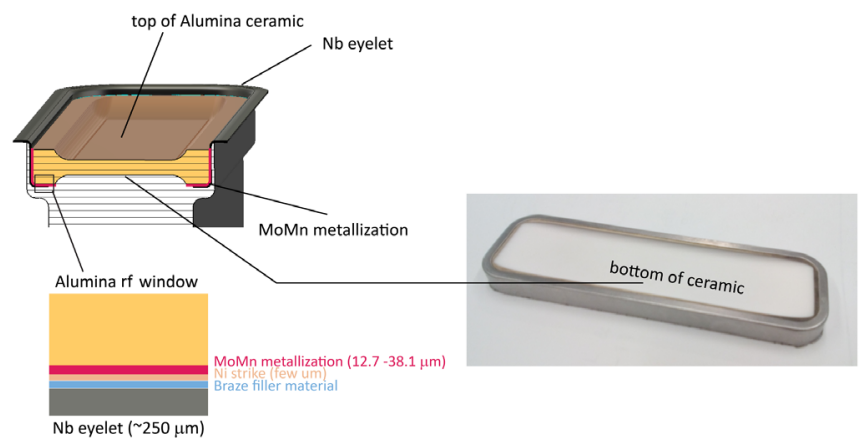

FIG. 5. Cross-sectional illustration (top left) and picture (right) of a standard cold rf ceramic windows as brazed to a $\mathrm{Nb}$ eyelet [1]. The bottom left figure illustrates the various layers of material involved. determines the volumetric losses $\left(\sim \pi f \tan \delta \int E^{2} d v\right)$ and depends strongly on the temperature. At L-band frequencies, the loss tangent is not exactly known. Rather sparse literature data suggest a value around $10^{-4}$ at room temperature [12], which may drop into the $10^{-6}$ and $10^{-7}$ range at $\mathrm{LHe}$ temperatures [13,14], which depends on the purity of the alumina apart from the frequency. The ceramic is brazed into a $\mathrm{Nb}$ eyelet ( $\sim 250 \mu \mathrm{m}$ thin), which is superconducting below the critical temperature of $\sim 9.2 \mathrm{~K}$ for $\mathrm{Nb}$. The $\mathrm{Nb}$ eyelet itself is electron beam welded to a relatively large $\mathrm{Nb}$ flange. The losses in the superconducting $\mathrm{Nb}$ material can be assumed negligible compared to normal conducting (nc) rf losses. The $\mathrm{nc}$ rf losses will arise due to the rf magnetic fields within the metallized areas of a ceramic.

Overall, both the losses in the metallization and the dielectric of a cold window have to be taken into account. Together with additional waveguide heat losses, this can lead to a reduction of cavity $Q_{0}$ values. One complication during cryomodule cavity acceptance or commissioning tests, when the $Q_{0}$ is measured as a function of $E_{\mathrm{acc}}$ for each cavity, is that one cannot readily differentiate between SRF losses in the $\mathrm{Nb}$ cavity walls and losses from the external circuit leaking into the LHe. Note that the metallization is a requirement for brazing the ceramic to the $\mathrm{Nb}$ eyelet for a UHV leak-tight bond. Traditionally and for decades, a molybdenum-manganese (50\%-70\% Mo, 5\%-10\% Mn) metallization with a nickel plating is a reliable technique for binding ceramics to metal, as outlined in Ref. [11].

\section{A. Assessment of electrical conductivity for the cold ceramic window metallization at $\mathrm{LHe}$ temperature}

A setup consisting of a double dogleg and window waveguide resonator is used at Jefferson Lab as a quality control tool for the cold windows to be installed on cavity pairs. The setup, shown in Fig. 6, employs a cold window with known losses to evaluate the yet unknown losses in a second cold window under test. The resonator is made from $\mathrm{Nb}$ and oscillates at $\sim 1.5 \mathrm{GHz}$ in the $\mathrm{TE}_{104}$ mode. This is a standing wave, while the coupling to the resonator is facilitated with two weakly coupled antennas, one on either end, to measure the quality factor in transmission. The inner conductors of the coupling antennas are made from $\mathrm{Nb}$ to minimize the losses in the external circuit, so that overall the dominant losses shall occur in the cold windows. A small pumping port is located in the middle of the straight central waveguide for evacuating the windowto-window enclosure, and one smaller pumping port on each coaxial output port for evacuating the volume of each coax-to-waveguide adapter formed between a window and a coaxial rf vacuum feedthrough. Waveguide flange connections are made with superconducting indium seals throughout. All connections must be UHV leak tight before immersing the setup in LHe. 


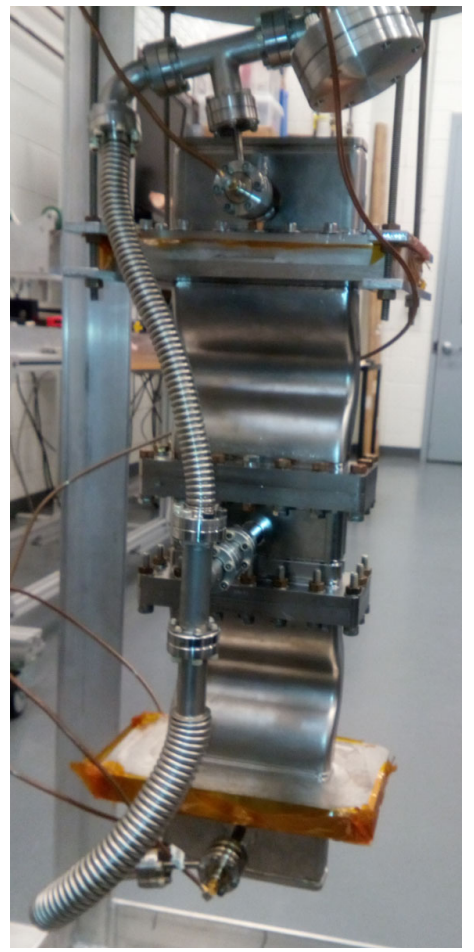

FIG. 6. Double dogleg and window waveguide resonator for characterizing rf losses of cold windows at LHe temperature in a vertical cryostat at low power value using a vector network analyzer. The top dogleg contains a reference cold window that is always employed as a reference, with known losses, against which a second cold window in a second dogleg is measured.

A cold window passes the test if the measured $Q_{0} \geq 28324$ of the resonator at a temperature of $\sim 4 \mathrm{~K}$. Based on an analytical computation of the rf field amplitude in a rectangular waveguide with the same cross section at a given forward power, this $Q_{0}$ is equivalent to $2 \mathrm{~W}$ of losses dissipated for a $5 \mathrm{~kW}$ forward traveling wave at $1.5 \mathrm{GHz}$. Note that this it is not an insignificant heat loss into the LHe bath, i.e., compared to the nominal SRF wall losses, though originally it had been deemed acceptable. In fact, per Table I above, the expected $Q_{0}$ degradation of cavities with cold windows tested in a vertical cryostat is on the order of $10 \%$. The average $Q_{0}$ value obtained from 62 doglegs and windows tested at $4.3 \mathrm{~K}$ between 2008 and 2016 is $30500 \pm 8464$.

Only a more recent improvement in numerical modeling, together with improved meshing capabilities, has provided a chance to more realistically estimate the electrical conductivity of the MoMn metallization in combination with the results from the cryogenic $\mathrm{TE}_{104}$ waveguide resonator. For this reason, the double dogleg and window waveguide resonator has been replicated as a numerical rf model in the software code CST Studio Suite [15] as depicted in Fig. 7 together with its mesh representation. The transparent view therein highlights the volume of the thin ceramic metallization volumes. The model includes the two coaxial coupling

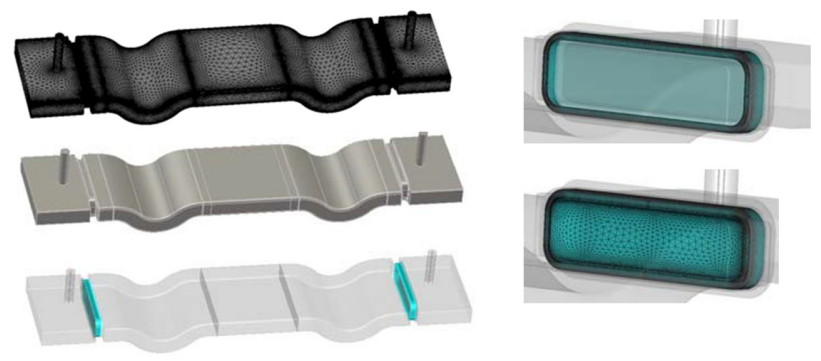

FIG. 7. Various views of the rf model and mesh of the double dogleg and window resonator. The overall number of tetrahedral mesh cells was $\sim 0.74 \mathrm{M}$ taking into account one symmetry plane.

ports. The small pumping port has been omitted for simplicity, which also allows utilizing one symmetry plane as a benefit. The model is meshed utilizing an anisotropic refinement of the tetrahedral mesh cells with curved elements of third order. The frequency of the $\mathrm{TE}_{104}$ mode obtained is 1494.0 MHz with $\epsilon_{r}=9.0$ assumed for the ceramics. This value is sufficiently close to measurement results, considering that fabrication tolerances for the resonator sheet metal production can as well change the frequency compared to an ideal model.

With this model and utilizing the average $Q_{0}(4 \mathrm{~K})=$ 30500 measured for cold windows in the same setup, the numerically equivalent conductivity for the MoMn metallization resulted in $\sigma(4 \mathrm{~K})=1.954 \times 10^{6} \mathrm{~S} / \mathrm{m}$. It is assumed that this value will not significantly improve when the window temperature is reduced to $2.07 \mathrm{~K}$, i.e., the nominal CEBAF cavity operating temperature. The computation took into account the resonator $\mathrm{Nb}$ wall losses assuming reasonable surface properties at a temperature of $4 \mathrm{~K}$. It shows that these losses are rather negligible compared to the cold window losses since yielding a corresponding $Q_{0}(4 \mathrm{~K})$ of $\sim 2 \times 10^{8}$. In a first evaluation, no dielectric losses have been taken into account. When assuming $\tan \delta=10^{-6}$, which is a rather conservative value, still $\sim 99.8 \%$ of the losses are originating in the metallization (with $Q_{0}=1.35 \times 10^{7}$ attributed to just the dielectric losses). The correction to the MoMn conductivity in the presence of dielectric losses is then rather small, yielding $\sigma(4 \mathrm{~K})=1.963 \times 10^{6} \mathrm{~S} / \mathrm{m}$. With this analysis, we have eventually obtained a realistic value for relevant metallization losses.

\section{EXPERIMENTAL RESULTS}

\section{A. Cavities tested in a vertical cryostat}

As part of the CEBAF cavity qualification process before helium tank installation, cavities are measured in cavity pairs fully immersed in $\mathrm{LHe}$ in a vertical dewar, with the LHe pumped to $2.07 \mathrm{~K}$. The $\mathrm{C} 75$ cavities for the first C75 cryomodule were characterized individually before cavitypair assembly, whereas C50 cavities were measured 


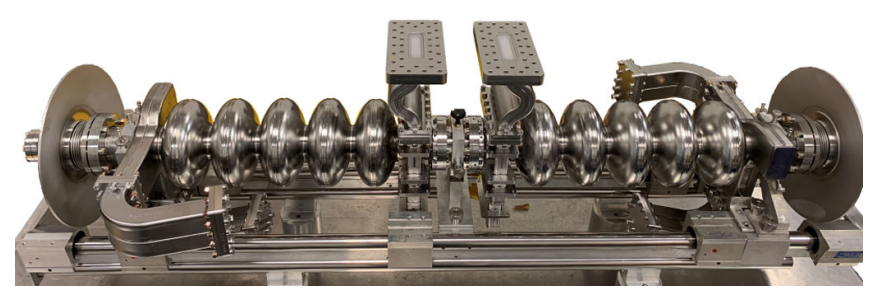

FIG. 8. C50 cavity pair assembled on a horizontal rail. The setup includes the doglegs with cold rf windows.

directly as cavity pairs. In any case, each cavity can be characterized individually, specifically scrutinizing the typical $Q_{0}\left(E_{\text {acc }}\right)$ curve. The potential presence of field emitters could require chemical reprocessing of a cavity interior in severe cases apart from high-pressure rinsing (HPR) with ultrapure water and drying and assembly in an ISO 5 clean room. The single-cavity configuration for a baseline cryogenic rf test consists of closing the FPC and high-order mode (HOM) waveguide ports with $\mathrm{Nb}$ blanks and using commercial rf vacuum feedthroughs with fixed-length $\mathrm{Cu}$ antennas to couple to the $\mathrm{TM}_{010}$ resonant mode through the beam line ports. One of the antennas is used as the input coupler, with $Q_{\text {ext,in }} \sim 1 \times 10^{10}$, and the other is used as a weakly coupled pickup antenna $\left(Q_{\text {ext,out }} \sim 1 \times 10^{12}\right)$.

A C50 cavity-pair assembly is shown in Fig. 8 as prepared for a test in one of the vertical cryostats in JLab's VTA depicting that each cavity is equipped with a dogleg housing the cold window. For this VTA configuration, special coax-to-waveguide adapters (top hats) made from $\mathrm{Nb}$, each equipped with a commercial rf vacuum feedthrough, are attached to the doglegs using indium seal connections. A top hat serves as the power input coupler port for a cavity, while a pickup antenna is positioned on the opposite end of a cavity on the HOM coupler waveguide, where a pickup port exists. A top hat increases the $Q_{\text {ext,in }}$ to $\sim 1 \times 10^{10}$, about 3 orders of magnitude higher than the $Q_{\text {ext }}$ of the FPC waveguide. This enables a VTA test at a sufficiently low power level, since the expected cavity $Q_{0}$ values are also on the order of $1 \times 10^{10}$. Besides doglegs, $\mathrm{Nb}$ elbows with HOM loads are also assembled onto each cavity in the cavity-pair configuration. A Nb inner adapter connects the two cavities, and an assembly with stainless steel end dishes is attached to the outer beam tube of each cavity.

The average low-field $\left(E_{\text {acc }} \sim 4 \mathrm{MV} / \mathrm{m}\right) Q_{0}$ value measured for nine $\mathrm{C} 75$ cavities, tested as individual cavities in the VTA at $2.07 \mathrm{~K}$, was $(13.4 \pm 1.8) \times 10^{9}$. The average low-field $Q_{0}$ value measured on eight C75 cavities tested as cavity pairs, with the dogleg housing the cold window, was $(10.6 \pm 1.2) \times 10^{9}$ [16]. The only treatment that was applied to the cavities after the individual test and before the cavity-pair assembly was HPR. The residual magnetic field during cooldown was monitored with cryogenic fluxgate magnetometers along the cavities, and it was $<1 \mu \mathrm{T}$

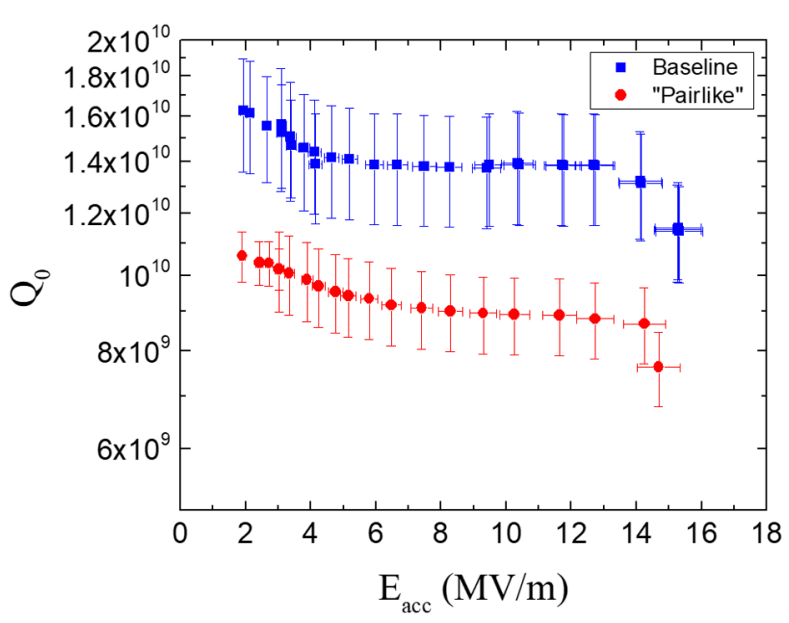

FIG. 9. VTA test results at $2.0 \mathrm{~K}$ for C50 cavity IA366 assembled in two configurations, i.e., without a dogleg and cold window (baseline) and with a dogleg and cold window ("pairlike").

for all of the tests. An example of the $Q_{0}\left(E_{\text {acc }}\right)$ curves measured at $2.07 \mathrm{~K}$ in a $\mathrm{C} 75$ cavity tested as individual cavity and as part of a cavity pair is shown in Fig. 1.

The average low-field $Q_{0}$ value measured for 20 C50 cavities tested as cavity pairs in the VTA at $2.07 \mathrm{~K}$ was $(9.4 \pm 2.6) \times 10^{9}$. No individual VTA measurements were performed for C20 and C50 cavities that would document the $Q_{0}$ degradation in reference to a cavity-pair test setup with a dogleg housing a cold window. One C50 cavity (IA366) was tested in a pairlike configuration with a dogleg or window attached after the baseline test was carried out as a "single-cavity" test setup. The $Q_{0}$ value at $E_{\text {acc }}=$ $4 \mathrm{MV} / \mathrm{m}$ achieved in the baseline test at $2.0 \mathrm{~K}$ is $(13.9 \pm 2.2) \times 10^{9}$, but it decreased to $(9.9 \pm 1.1) \times 10^{9}$ at a similar low field once the dogleg with a cold window was assembled to the cavity. The $Q_{0}$ degradation due to the cold window losses is, therefore, as high as $40 \%$. The $Q_{0}$ value in this configuration was also measured at $2.07 \mathrm{~K}$ and resulted in $(8.9 \pm 1.0) \times 10^{9}$ at a low field. $Q_{0}\left(E_{\text {acc }}\right)$ curves measured for cavity IA366 are shown in Fig. 9. The residual magnetic field in both tests was $<0.3 \mu \mathrm{T}$.

\section{B. Cavities tested in CEBAF cryomodules}

Cavities installed in CEBAF cryomodules are fully equipped and driven by individual klystrons for $\mathrm{CM}$ commissioning tests in the tunnel before beam operation. Connection of the klystron output to the cavities occurs through rectangular waveguides. Since a rectangular FPC waveguide by design has no means for changing the external $Q, Q_{\text {ext FPC }}$, it can be adjusted only within limits by a waveguide stub tuner. The nominal $Q_{\text {ext FPC }}$ value of each cavity is typically achieved during production cavity postprocessing by mechanical deformation of the FPC waveguide body. This is done on a cavity bench with a specific tool [11]. The CEBAF stub tuners are installed in 


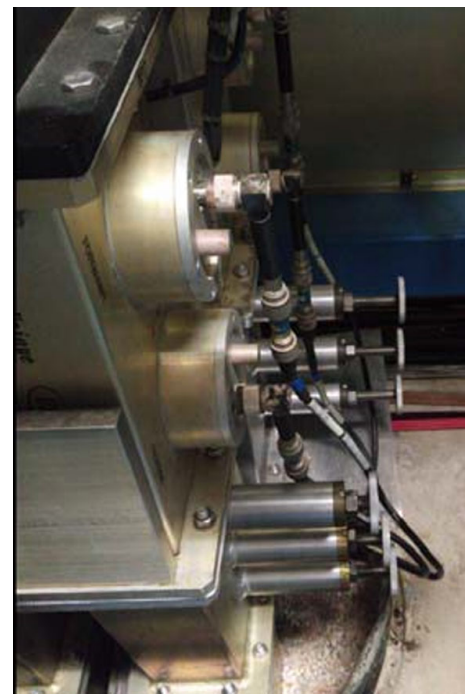

FIG. 10. Picture of WR650 three-plunger stub tuners accessible in the CEBAF service buildings within klystron cabinets.

the WR650 $(16.51 \mathrm{~cm} \times 8.26 \mathrm{~cm})$ waveguide and are located within the klystron cabinets (see Fig. 10). A stub tuner consists of three manually adjustable plungers penetrating the waveguide. It allows changing the $Q_{\text {extFPC }}$ value within limits. The penetration of the plungers will inevitably create a resonant circuit between a cavity and a stub tuner that, in turn, may alter the losses at the cold windows. Three waveguide stub tuner models are utilized in CEBAF due to specific space limitations in the klystron cabinets. Note that rf breakdowns can occur if the plunger is inserted too far into the waveguide, limiting the $Q_{\text {ext FPC }}$ adjustability.

The standard method for measuring the rf heat load of a cavity in a cryomodule at JLab is the pressure rise method [17], which follows this procedure. (1) Close the CM He supply and return valves. (2) Measure the He bath pressure versus time over a $20 \mathrm{~s}$ interval and obtain $\frac{d P}{d t}$ from a leastsquares linear curve fit, $\left.\frac{d P}{d t}\right|_{\text {static } 0}$. (3) Turn the heater on with fixed, known power $P_{\text {heater }}(\sim 20 \mathrm{~W})$ and measure $\left.\frac{d P}{d t}\right|_{\text {heater }}$. (4) Turn the heater off and measure $\left.\frac{d P}{d t}\right|_{\text {static } 1}$. (5) Turn the rf on and measure $\left.\frac{d P}{d t}\right|_{r f}$. (6) Turn the rf off and measure $\left.\frac{d P}{d t}\right|_{\text {static } 2}$.

The rf heat load $P_{r f}$ is then calculated as

$$
P_{r f}=\frac{\left.\frac{d P}{d t}\right|_{r f}-\left.\frac{d P}{d t}\right|_{\text {static } 2}}{\left.\frac{d P}{d t}\right|_{\text {heater }}-\left.\frac{d P}{d t}\right|_{\text {static } 1}} P_{\text {heater }} \text {. }
$$

The typical pressure rise is of the order of $2 \mathrm{mTorr} / \mathrm{s}$. Because $P_{r f}$ is measured at different temperatures, a temperature correction (typically $\lesssim 5 \%$ ) is applied to calculate $P_{r f}$ at $2.07 \mathrm{~K}$ :

$$
P_{r f}(2.07 \mathrm{~K})=\frac{Q_{0}(T) P_{r f}(T)}{Q_{0}(2.07 \mathrm{~K})},
$$

where $Q_{0}(T)$ is calculated as

$$
Q_{0}(T)=\frac{E_{\mathrm{acc}}^{2}}{k^{2} P_{r f}(T)}
$$

$k=4.26 \mathrm{MV} /(\mathrm{m} \sqrt{J})$ is a constant determined by the cavity geometry, and $P_{r f}$ is calculated from Eq. (1). $E_{\text {acc }}$ is obtained as

$$
E_{\mathrm{acc}}=k \sqrt{\frac{Q_{\mathrm{ext}, \mathrm{out}} P_{t}}{2 \pi f_{0}}},
$$

where $f_{0}$ is the cavity resonant frequency and $Q_{\text {ext,out }}$ is a fixed value obtained from an emitted power measurement with a pulsed rf at low field. $Q_{0}(2.07 \mathrm{~K})$ is calculated as

$$
\begin{aligned}
Q_{0}(2.07 \mathrm{~K}) & =\frac{G}{R_{s}(2.07 \mathrm{~K})} \\
& =\frac{Q_{0}(T)}{1+\frac{Q_{0}(T)}{G} A\left(e^{-\Delta /(2.07 \mathrm{~K})}-e^{-\Delta / T}\right)},
\end{aligned}
$$

where $R_{s}$ is the surface resistance, $G \simeq 275 \Omega$ is the geometry factor, $A=3.274 \times 10^{-5} \Omega$ is the preexponential factor in the approximate expression of the $\mathrm{Nb}$ BardeenCooper-Schrieffer surface resistance, $R_{s} \approx A e^{-\Delta / T}$, and $\Delta=15.486 \mathrm{~K}$ is the gap value for $\mathrm{Nb}$. The measured $\mathrm{rf}$ heat load is the sum of the power dissipated on the cavity walls and any other losses, such as heating of the cold window or from the FPC waveguide section below the $50 \mathrm{~K}$ thermal intercept $P_{w}$, which are dissipated in the He bath. The rf heat load as a function of the klystron forward power $P_{f}$ and detuning angle $\phi$ is given by

$P_{c}+P_{w}=\frac{4 P_{f} \beta}{\left[1+(\tan \phi)^{2}\right](1+\beta)^{2}} \simeq \frac{4 P_{f}}{\beta\left[1+(\tan \phi)^{2}\right]}$,

where $\beta \gg 1$ is the coupling factor of the FPC. By measuring $P_{f}(\phi)$ at a fixed $E_{\text {acc }}$ (constant $P_{c}$ ), the standing-wave pattern is changed in the FPC waveguide, resulting in different locations of the peak fields. Dividing Eq. (6) by $P_{c}$ results in the following ratio $P_{w 0} / P_{c}$ at $\phi=0$, where the subscript 0 denotes the value of a variable at $\phi=0$ :

$$
\frac{P_{w 0}}{P_{c}}=\frac{4 P_{f}}{\beta}-1
$$

Given that $P_{r f 0}=P_{c}+P_{w 0}, P_{c}$ and $P_{w 0}$ can be calculated from measured quantities as 


$$
P_{c}=\frac{P_{\mathrm{rf} 0}}{2 P_{f 0}} \frac{\pi f_{0}\left(E_{\mathrm{acc}} / k\right)^{2}}{Q_{\mathrm{ext} \mathrm{FPC}}}
$$

and

$$
P_{w 0}=P_{\text {rfo }}-P_{c}
$$

respectively, where $Q_{\text {ext FPC }}$ is measured from the decay time during a pulsed rf measurement.

\section{Measurements of cavities in cryomodule FELO4}

The rf heat load as a function of the detuning angle was measured for cavities 1-6 of cryomodule FEL04, which was installed in the FEL vault in 2016. The FEL04 cryomodule was a C20 style, without doglegs. Figure 11 shows a plot of $P_{r f}$ at $2.07 \mathrm{~K}$ as a function of the detuning angle for cavities 1-6 while maintaining the accelerating gradient at $7 \mathrm{MV} / \mathrm{m}$. The forward power increased monotonically with increasing detuning angle between $\sim 2-6 \mathrm{~kW}$ for $\phi=0^{\circ}-50^{\circ}$. Each data point in Fig. 11 is the average of three measurements under the same conditions.

Figure 12 shows a plot of the rf heat load at $2.07 \mathrm{~K}$, $E_{\text {acc }}=7 \mathrm{MV} / \mathrm{m}$ and $\phi=0^{\circ}$ as a function of $Q_{\text {extFPC}}$. Figure 13(a) shows a plot of the rf heat load at $2.07 \mathrm{~K}$ measured in cavities 4-6 in the detuned state, such that all of the rf heat load is due to dissipation in the FPC waveguide and cold window, as a function of the forward power. Cavity 4 has the highest rf heat load and exhibited notable waveguide vacuum activity during the measurements. Figure 13(b) shows a plot of $P_{r f}$ at $2.07 \mathrm{~K}$ for a detuned cavity and $P_{f} \sim 2.5 \mathrm{~kW}$ versus $P_{\text {rfo }}$ at $2.07 \mathrm{~K}$, $E_{\text {acc }}=7 \mathrm{MV} / \mathrm{m}$ (for which $P_{f}=2-2.5 \mathrm{~kW}$ ), showing a clear indication that the higher rf heat load, which would be

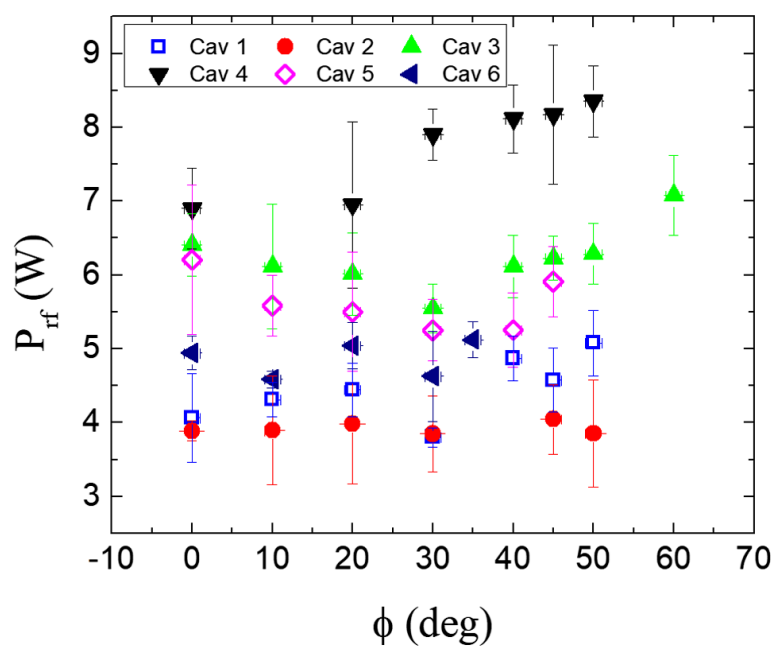

FIG. 11. The rf heat load at $2.07 \mathrm{~K}$ and $E_{\text {acc }}=7 \mathrm{MV} / \mathrm{m}$ measured as a function of the detuning angle for cavities 1-6 in C20 CM FEL04.

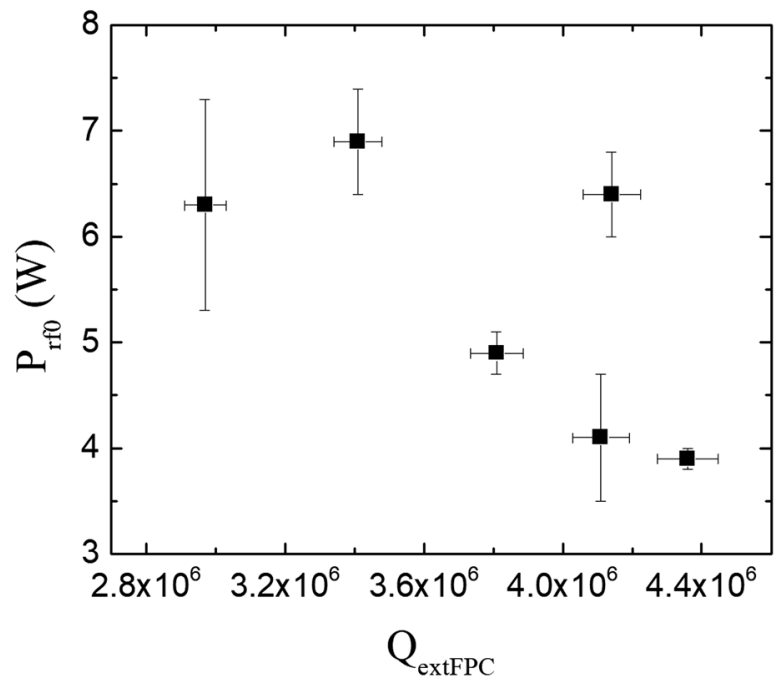

FIG. 12. The rf heat load at $2.07 \mathrm{~K}$ and $7 \mathrm{MV} / \mathrm{m}$ at $\phi=0^{\circ}$ as a function of the $Q_{\text {extFPC }}$ for cavities in FEL04.
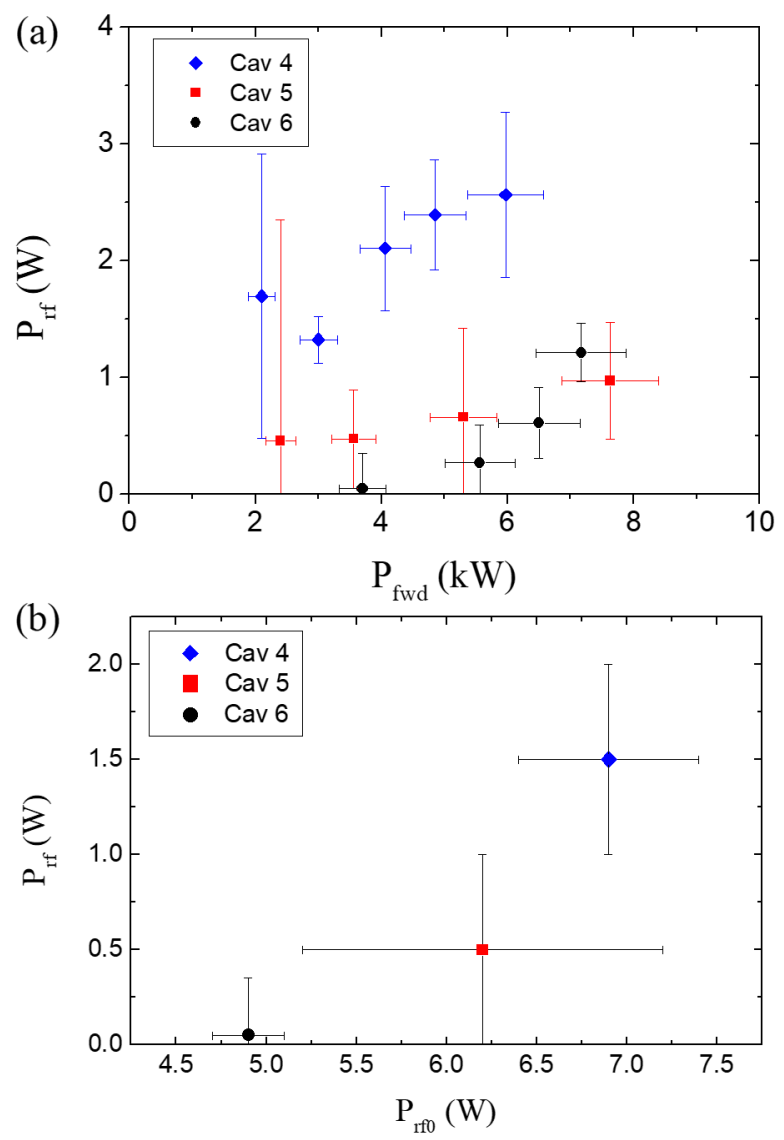

FIG. 13. The rf heat load at $2.07 \mathrm{~K}$ in the case of fully detuned cavities, as a function of the forward power measured in C20 CM FEL-04 (a) and rf heat load of fully detuned cavities for $P_{f} \sim$ $2.5 \mathrm{~kW}$ as a function of the rf heat load with the cavities on crest $\left(\phi=0^{\circ}\right)$ at $7 \mathrm{MV} / \mathrm{m}(\mathrm{b})$. 
interpreted as a cavity with lower $Q_{0}$, is, in fact, due to higher losses in the FPC waveguide and/or cold window.

Equations (8) and (9) were used to deconvolute the cavity losses from FPC waveguide losses from the total $\mathrm{rf}$ heat load. The average wall-loss dissipation of the six cavities at $7 \mathrm{MV} / \mathrm{m}$ was $P_{c}=(4 \pm 1) \mathrm{W}$, and the average dissipation from the FPC waveguide and cold window is $P_{w 0}=(1.2 \pm 0.5) \mathrm{W}$, indicating that more than $20 \%$ of the total heat load is due to losses external to the cavity surface.

\section{Measurements of cavities in cryomodule 1L13}

Measurements of the rf heat load as a function of the detuning angle were carried out for cavities 1,2, 4, and 8 of cryomodule 1L13 during its commissioning test in CEBAF in November 2017. Cavities 1 and 2 were the two prototype C75 cavities built at JLab, whereas the remaining cavities are C50 style. The remanent magnetic field at the cavities' location was mitigated in this CM by degaussing some of its components and by installing an additional magnetic shield close to each cavity, inside the He vessels. Figure 14 shows a plot of $P_{r f}$ at $2.07 \mathrm{~K}$ as a function of detuning angle while maintaining the accelerating gradient at $7 \mathrm{MV} / \mathrm{m}$. The forward power was less than $2 \mathrm{~kW}$ for $\phi=$ $0^{\circ}-50^{\circ}$ because of the higher $Q_{\text {ext FPC }}$ for these cavities compared to those in FEL04. Each data point in Fig. 14 is the average of three measurements under the same conditions. Stub tuners are installed in the waveguide for each of these cavities, but the position of the stubs was unknown at the time of the measurements. The data for cavity 8 showed that the rf heat load is reduced by $\sim 40 \%$ by simply increasing the detuning angle. Measurements of the rf heat load at $2.07 \mathrm{~K}$ for fully detuned cavities as a function of the forward power for the four cavities tested in 1L13 showed

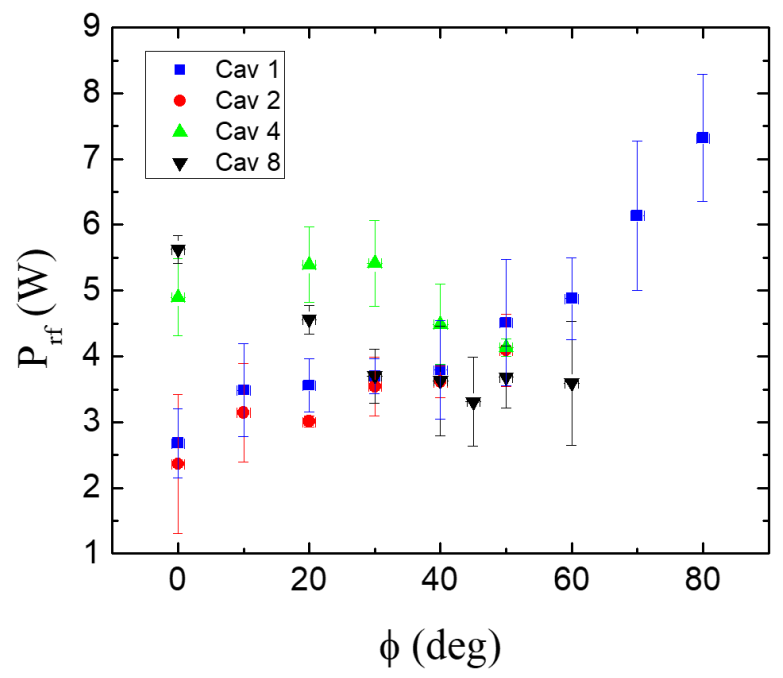

FIG. 14. The rf heat load at $2.07 \mathrm{~K}$ and $7 \mathrm{MV} / \mathrm{m}$ measured as a function of the detuning angle for four cavities in C50 CM 1L13. Cavities 1 and 2 are C75 prototype cavities. that losses due to the FPC waveguide and/or cold window could be between $0.7-1.4 \mathrm{~W}$ per kilowatt of forward power.

Heat load measurements were repeated in August 2018 on cavities 4 and 8 for different plunger positions of their stub tuners, resulting in different values of the $Q_{\text {ext FPC }}$, the results being shown in Fig. 15. The data clearly show that the rf heat load in the on-crest condition $\left(\phi=0^{\circ}\right)$ changed by up to $\sim 40 \%$ with different stub tuner settings.

Figure 16 summarizes $P_{r f 0}$ at $2.07 \mathrm{~K}$ and $E_{\text {acc }}=$ $7 \mathrm{MV} / \mathrm{m}$ as a function of the $Q_{\text {ext FPC }}$, showing lower rf heat loads for the $\mathrm{C} 75$ cavities. The rf heat load of the two C75 cavities is consistent with the average rf heat load of $(2.6 \pm 0.2) \mathrm{W}$ measured for eight $\mathrm{C} 75$ cavities in the first C75 CM, recently tested at JLab's Cryomodule Test Facility. C75 cavities by design have a larger distance between the end cell and the FPC waveguide than the C20 and $\mathrm{C} 50$ cavities. This consequently results in a larger $Q_{\text {extFPC }}$ and a lower $P_{f}$ for the same stored energy (same $\left.E_{\text {acc }}\right)$. Hence, to first order we can assume that the rf losses scale with the ratio of the $Q_{\text {ext FPC }}$ values if one does not account for standing-wave effects. A factor of 2.5 larger $\mathrm{rf}$
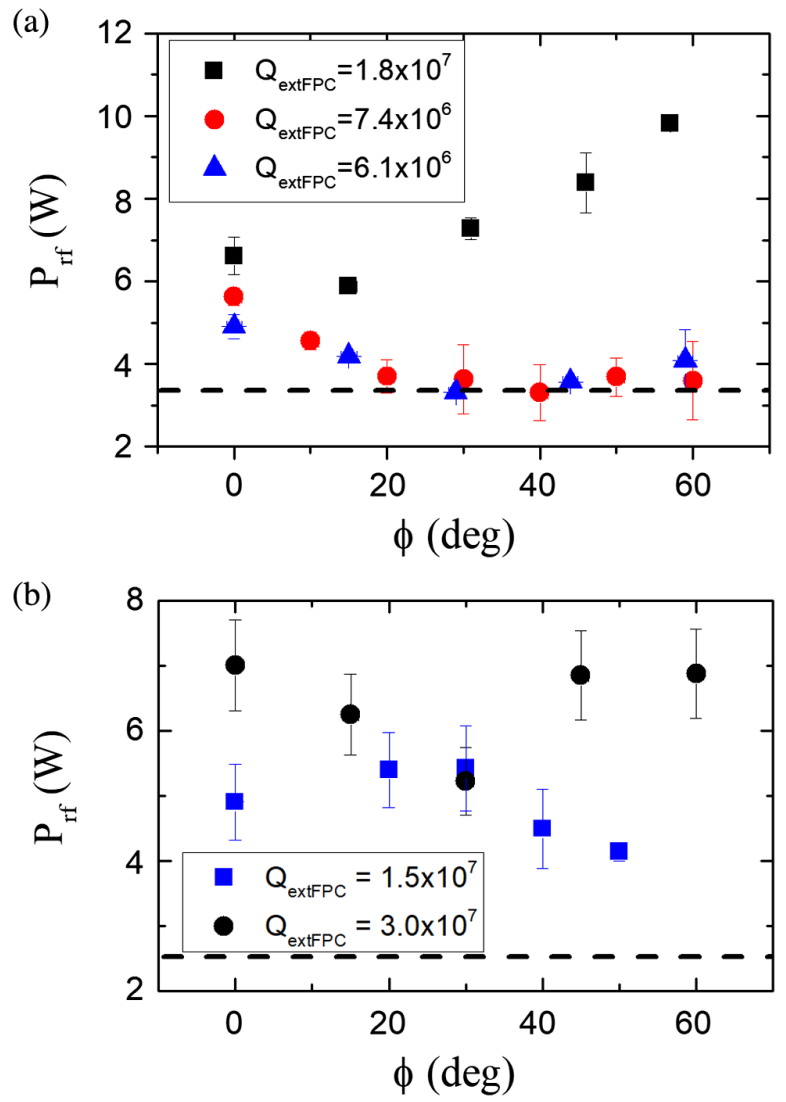

FIG. 15. The rf heat load at $2.07 \mathrm{~K}$ and $7 \mathrm{MV} / \mathrm{m}$ as a function of the detuning angle for different stub tuner settings, resulting in different $Q_{\text {extFPC }}$ values for cavity 1L13-4 (a) and 1L13-8 (b). The dashed lines represent the rf heat load measured at the same $E_{\text {acc }}$ value in the VTA test of each of these cavities, tested as cavity pairs. 


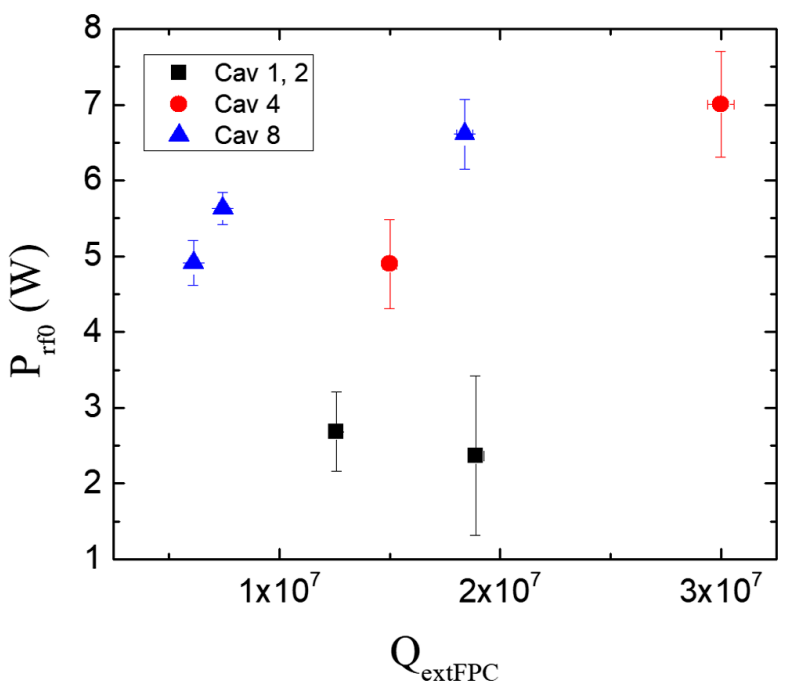

FIG. 16. The rf heat load at $2.07 \mathrm{~K}, 7 \mathrm{MV} / \mathrm{m}$, and $\phi=0^{\circ}$, as a function of $Q_{\text {ext FPC }}$ for cavities measured in C50 CM 1L13. Cavities 1 and 2 are prototype C75 cavities installed in 1L13.

losses would, therefore, be anticipated in C50 versus C75 cavities when assuming the nominal $Q_{\text {extFPC }}$ values (cf. Table I). In the case of Fig. 16, though, differing stub tuner settings were in place, resulting in differing $Q_{\text {ext FPC }}$ values. As a reference, the $P_{c}$ values at $E_{\text {acc }}=7 \mathrm{MV} / \mathrm{m}$, at the nominal $Q_{0}$ values listed in Table I, are 2.8 and $3.8 \mathrm{~W}$ for C75 and C50 cavities, respectively. $P_{r f 0}$ measured for the $\mathrm{C} 75$ cavities is consistent with such a value; however, it exceeds the estimate by $31 \%$ minimally and up to $84 \%$ for C50 cavities. This result suggests that the rf heat load for C50 cavities more strongly depends on the actual standingwave amplitude (thus, stub tuner setting) in the transmission line determining the $Q_{\text {ext FPC }}$ and on the individual cold window losses, that may vary to some extent as experimentally observed in the double dogleg and window resonator.

\section{NUMERICAL SIMULATIONS}

\section{A. VTA cavity-pair configuration}

In a VTA cavity-pair configuration, the excitation of the $\mathrm{TM}_{010} \pi$ mode will result in standing waves within the waveguide between the top hat and the FPC stub. Consequently, elevated rf losses in the cold window can arise compared to a single-cavity test setup without a cold window. In this case, the $Q_{0}$ value of a cavity can degrade already. This setup is yet not fully identical with that of a cavity in a cryomodule and when much higher power levels are needed.

To better understand potential cold window losses in a cavity-pair test configuration, the setup has been mimicked numerically. Yet, only one cavity is required for this purpose (pairlike configuration). Figure 17 shows the corresponding rf model and its mesh representation,

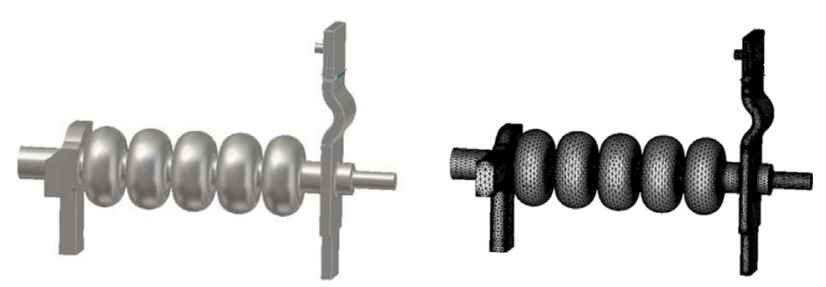

FIG. 17. The rf model (left) and mesh representation (right) for simulating a C75 cavity in a VTA cavity-pair configuration.

respectively, for C75 cavities. Similar mesh refinement settings have been chosen as for the $\mathrm{TE}_{104}$ resonator, though this model is significantly larger and stricter numerical limitations apply. For ease of meshing, the elbows representing the HOM waveguide ends were omitted. This does not significantly impact results for any fundamental passband mode, since the waveguide cutoff is at $\sim 1.9 \mathrm{GHz}$. A model of the cryogenic $\mathrm{rf}$ feedthrough attached to the top hat coaxial port has actually been included, though not shown in Fig. 17. Attached to the rf feedthrough is a coaxial absorber well matched at $1.5 \mathrm{GHz}$ (also not shown) so that the eigenmode solver delivers a complex solution, i.e., allowing for a traveling mode in the coaxial line as is the case in reality for a matched condition at the coaxial line.

The $\mathrm{TM}_{010} \pi$ mode frequency obtained numerically is $f_{0}=1496.85 \mathrm{MHz}$ in vacuum. Note that the field-flatness (FF) specification for $\mathrm{C} 75$ cavities is to yield $\geq 95 \%$ after final bench tuning. It is important to mention that a similar FF was obtained numerically. This high FF is a prerequisite for a proper assessment of the cold window losses, i.e., to resemble a realistic coupling of the cavity rf fields to the FPC waveguide fields responsible for the window losses. Eventually, the external $Q$ has been computed. This has been done by evaluating the volume loss in the coaxial absorber. It also depends on the actual (custom) length of the copper input antenna, which is attached to the vacuum side of the rf feedthrough. In reality, the antenna is almost flush with the top hat inner waveguide surface. For the numerical model with a flush antenna, the result is $Q_{\text {ext,in }}=2.69 \times 10^{10}$, which is sufficiently close to the average $Q_{\text {ext,in }}=2.13 \times 10^{10}$ measured for C75 cavities.

An rf model for C50 cavities in a "pairlike" configuration was also built, similarly to that for the $\mathrm{C} 75$ cavities. One complication arises here since a $\mathrm{C} 20$ or $\mathrm{C} 50$ cavity with end groups will not result in a sufficiently flat field $(\mathrm{FF} \approx 92 \%)$ as designed, while the $\mathrm{TM}_{010} \pi$ mode frequency is only $\sim 1489.5$ (1489.0) $\mathrm{MHz}$ in vacuum (air). In reality, this implies that the original $\mathrm{C} 20$ cavities as produced by industry required significant bench tuning via plastic cell deformations to meet both the FF and warm target frequency specification $(\sim 1494 \mathrm{MHz})$. For the rf model, the end cell adjacent to the FPC end group was slightly elongated at the equator to improve the field flatness to a sufficiently high value of $97.4 \%$. The $\pi$ mode frequency 
TABLE II. Numerically computed $Q$ values of individual components as present in a C50 and C75 cavity-pair VTA test configuration.

\begin{tabular}{lcccc}
\hline \hline Component & Material & $\sigma(\mathrm{S} / \mathrm{m})$ or tan $\delta$ at $2.07 \mathrm{~K}$ & $Q_{i}$ for C50 cavity & $Q_{i}$ for C75 cavity \\
\hline SRF cavity interior wall & $\mathrm{Nb}$ & $1.4 \times 10^{19}$ & $1.3 \times 10^{10}$ & $1.3 \times 10^{10}$ \\
rf feedthrough vacuum side antenna & $\mathrm{Cu}$ & $5.63 \times 10^{10}$ & $7.5 \times 10^{13}$ & $5.0 \times 10^{14}$ \\
rf feedthrough inner or outer conductor & $304 \mathrm{SS}$ & $2.02 \times 10^{6}$ & $4.7 \times 10^{11}$ & $2.9 \times 10^{12}$ \\
rf feedthrough vacuum window & $\mathrm{Al}_{2} \mathrm{O}_{3}$ & $\tan \delta=3.09 \times 10^{-6}$ & $>1 \times 10^{12 \mathrm{a}}$ & $1.0 \times 10^{14}$ \\
rf feedthrough, alumina sleeves & $\mathrm{Cupronickel}$ & $2.94 \times 10^{6}$ & $2.3 \times 10^{12}$ & $1.5 \times 10^{13}$ \\
Cold window metallization & $\mathrm{MoMn}$ & $1.96 \times 10^{6}$ & $8.8 \times 10^{9}$ & $9.6 \times 10^{10}$ \\
& & $3.37 \times 10^{6}$ & $>1 \times 10^{12 \mathrm{a}}$ & $1.6 \times 10^{13}$ \\
\hline
\end{tabular}

${ }^{\mathrm{a}}$ The computation combined the losses for both ceramics, resulting in $Q=1.11 \times 10^{12}$.

computed for the cavity with this FF is $1489.28 \mathrm{MHz}$ in vacuum. For the sake of evaluating the cold window losses, the frequency can be deemed sufficiently close to the cold vacuum target frequency of $\sim 1497 \mathrm{MHz}$ without requiring one to further modify every single cell numerically. Note that the rf feedthrough antenna length was adjusted to yield $Q_{\text {ext,in }}=4.32 \times 10^{9}$ at the coaxial input coupler, in order to match the measured value. Compared to the C75 cavity design, the FPC waveguide is located much closer to the cavity end cell due to the desired lower $Q_{\text {ext FPC }}$ by design. Consequently, this will elevate the field amplitude in the FPC at the same stored energy so that larger cold window losses can already be expected than in C75 cavities.

The CST eigenmode solver generally computes the rf field at $1 \mathrm{~J}$ stored energy, $W_{s}$, and provides the power losses for individual components, $P_{i}$, that can be assigned to different materials, for which, in turn, different electrical conductivities can be assumed. This results in individual $Q$ values according to $Q_{i}=2 \pi f_{0} W_{s} / P_{i}$ as summarized in Table II. Herein, the $\mathrm{Nb}$ surface conductivity was adjusted to yield a total cavity intrinsic $Q$ value $\left(Q_{0}\right)$ that agrees with the average measured $Q_{0}$ from the single-cavity tests. The conductivity of the MoMn was taken to be $1.96 \times 10^{6} \mathrm{~S} / \mathrm{m}$, as discussed in Sec. II A, for the C 75 cavity, whereas $3.37 \times 10^{6} \mathrm{~S} / \mathrm{m}$ was used for the C50 cavity to match the quality factor of the specific dogleg or window used with cavity IA366. Other nc metals arising within the top hat $\mathrm{rf}$ feedthrough have been assigned with similarly low electrical conductivities $\left(\sim 2 \times 10^{6} \mathrm{~S} / \mathrm{m}\right.$ at $2.07 \mathrm{~K}$, except for the copper antenna assumed to be in the mid- $1 \times 10^{10} \mathrm{~S} / \mathrm{m}$ range at $2.07 \mathrm{~K}$ ), yet their $Q_{i}$ values are all significantly higher than that of the cold window metallization. The loss tangents of the alumina ceramics have been assumed to be $\tan \delta=3.09 \times 10^{-6}$. This is a factor of 10 higher than what has been measured at $4 \mathrm{~K}$ for a high-purity, polycrystalline $\mathrm{Al}_{2} \mathrm{O}_{3}$ at $7.53 \mathrm{GHz}$ [14], thus deemed a conservative value. The corresponding $Q_{i}$ values are yet not significant, e.g., $1.0 \times 10^{14}$ for the cold window and $1.6 \times 10^{13}$ for the feedthrough ceramic, which has only a 92\%-98\% purity, though [18].

The results shown in Table II indicate that rf losses from the MoMn are the dominant contribution to the heat load for both C75 and C50 cavities, accounting for $\sim 12 \%$ and $\sim 65 \%$ of the $Q_{0}$ reduction for C75 and C50 cavities, respectively. The $Q_{0}$ reduction calculated for the C50 cavity is $\sim 30 \%$ larger than measured, but it demonstrates that the cold window plays a crucial role concerning the mechanism of the $Q_{0}$ degradation. The observed discrepancy from the experimental results could have several other reasons. One arises from actual fabrication tolerances for both the cells and the end groups including the cavity to FPC waveguide separation. These can alter the actual coupling strength between the cavity and the FPC waveguide, which, for instance, is evidenced by variations of $Q_{\text {ext FPC }}$ values of cavities measured on a bench as delivered by industry. Furthermore, by design, the $Q_{\text {extFPC }}$ is in the lower $1 \times 10^{6}$ range for a $\mathrm{C} 20$ cavity. The waveguide body of the FPC close to the cavity is, therefore, mechanically tuned at JLab to reach the specified $Q_{\text {extFPC }}\left(6.6 \times 10^{6}\right)$, which is elevated slightly again (to $8 \times 10^{6}$ ) for refurbished C50 cavities. In either case, the $Q_{\text {ext FPC }}$ is adjusted by a plastic deformation of the broad wall of the FPC waveguide stub close to the cavity beam tube. The impact on results due to a deformed waveguide body could not be accounted for in numerical simulations and, since it depends on an asbuilt and as-tuned cavity, might vary from cavity to cavity. Note that millimeter deviations from nominal values are not uncommon for sheet metal production, and even weld shrinkages due to electron beam welding can be on the order of a millimeter. One also should consider that industrial fabrication and quality-control standards have continuously improved over the recent decades after the original C20 cavities were built, and this leaves open a lot of questions concerning the fabrication accuracy of C20 cavity cells and end groups, which can alter the cold window losses to an unknown extent.

\section{B. Cryomodule configuration}

Numerical simulations have been conducted to resemble the cavity cryomodule setup including a three-plunger stub tuner. The rf model is shown in Fig. 18. It includes a straight stub tuner placed at some distance away from the cavity in the narrow transmission line above the cold 


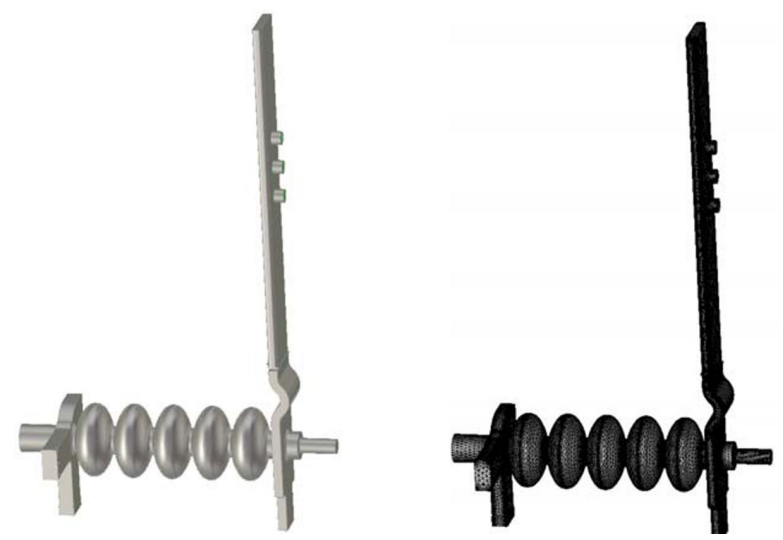

FIG. 18. The rf model (left) and mesh representation (right) for simulating a $\mathrm{C} 50$ cavity in a CM configuration.

window. The warm window has not been included to simplify the model and to focus solely on the cold window losses. A field flatness of $97.4 \%$ and a $\pi$ mode frequency of $f_{0}=1489.28 \mathrm{MHz}$ in vacuum was achieved in the CM configuration as was the case for the VTA cavity-pair configuration of the C50 model. These results are not significantly affected by the chosen plunger penetration. Note that the eigenmode solver implies no detuning angle $\left(\phi=0^{\circ}\right)$.

Except for the waveguide size, the three-plunger stub tuner model resembles the dimensions of an actual straight WR650 stub tuner. An rf model accounting for the entire power transmission line including the transition to a WR650 waveguide and a WR650 stub tuner is conceivable but will result in a significantly larger number of mesh cells. This is not necessary, since the rf model allows creating similar standing-wave scenarios as in reality by changing the penetration of each plunger independently.

The penetration of any single plunger from the inner long waveguide wall should not exceed the waveguide inner height, which is $24.5 \mathrm{~mm}$ above the dogleg or cold window. In the following, we use the nomenclature $(i, j, k)$ to denote the penetration of each plunger (in units of centimeters) referred to the inside of the waveguide wall, while the sequence is chosen from the plunger closest to the plunger farthest away from the cavity. For example, the plunger positions $(0,0,0)$ denote that all plungers are flush with the waveguide wall. A negative value would imply a retraction of a plunger into a stub tuner port, which is possible up to a
TABLE IV. Computed rf heat load at $E_{\text {acc }}=7 \mathrm{MV} / \mathrm{m}$ for C50 and $\mathrm{C} 75$ cavities in CM configuration, assuming a combined $Q_{0}$ as numerically assessed for flush plunger positions.

\begin{tabular}{lcc}
\hline \hline Cavity type & $Q_{0}(2.07 \mathrm{~K})$ & $P_{r f 0}(\mathrm{~W})$ \\
\hline C50 & $3.9 \times 10^{9}$ & 6.5 \\
C75 & $1.1 \times 10^{10}$ & 2.0 \\
\hline \hline
\end{tabular}

certain limit with the actual tuner mechanism. Normal conducting losses in the metallic stub tuner plungers are neglected. Note that rf waves reflected from a cavity would be directed toward the rf circulator and into its water-cooled load, which protects each klystron. However, the CEBAF circulators are not necessarily well-matched devices at $1497 \mathrm{MHz}$, and they are rf phase and temperature dependent. For the rf model, the transmission line above the stub tuner has been terminated with a waveguide absorber (not shown) that yields $S_{11} \sim 20 \mathrm{~dB}$ at the resonant frequency rather than assuming a better-matched absorber, e.g., with $S_{11}<30 \mathrm{~dB}$ that could be readily achieved. This seems to be more realistic given the discussion above.

A similar computation has been done for a C75 CM configuration, and the results are summarized in Table III for both $\mathrm{C} 50$ and $\mathrm{C} 75$ cavities with flush plunger positions. The computed $Q_{\text {extFPC }}$ for each type of cavity are in agreement with the design values. Table IV summarizes the relevant results for both the $\mathrm{C} 50$ and $\mathrm{C} 75 \mathrm{CM}$ configurations with flush plunger positions: The combined $Q_{0}$ is as low as $3.9 \times 10^{9}$ for the C50 cavity configuration, whereas it is $1.1 \times 10^{10}$ for the C75 cavity configuration. Compared to the pure cavity $Q_{0}$ at the same temperature, this means that the C50 cavity suffers a $Q_{0}$ degradation of $\sim 70 \%$. For the $\mathrm{C} 75$ cavity, this degradation is, however, reduced to $\sim 16 \%$. These results are independent from $E_{\text {acc }}$. The rf heat loads in Table IV have been computed at $E_{\text {acc }}=$ $7 \mathrm{MV} / \mathrm{m}$ and are consistent with the results from CM measurements shown in Fig. 16. It, therefore, becomes obvious that a C75 cavity by design is less prone to suffer from cold window losses as C50 cavities thanks to the higher $Q_{\text {extFPC }}$ value.

The flush plunger positions result in a negligible standing wave at the cold window location compared to the forward traveling wave component. To investigate the effect on the rf heat losses in the presence of more

TABLE III. Numerically computed $Q$ values of individual components as present in a C50 and C75 cavity in the $\mathrm{CM}$ configuration with plunger positions $(0,0,0)$. There was no discernable difference in the total volumetric rf losses whether or not the dielectric losses were included as part of the computation.

\begin{tabular}{lcccc}
\hline \hline Component & Material & $\sigma(\mathrm{S} / \mathrm{m})$ at $2.07 \mathrm{~K}$ & $Q_{i}$ for C50 cavity & $Q_{i}$ for C75 cavity \\
\hline SRF cavity interior wall & $\mathrm{Nb}$ & $1.4 \times 10^{19}$ & $1.3 \times 10^{10}$ & $1.3 \times 10^{10}$ \\
Cold window metallization & MoMn & $1.96 \times 10^{6}$ & $5.6 \times 10^{9}$ & $7.1 \times 10^{10}$ \\
$Q_{\text {ext FPC }}$ & Matched absorber & & $1.7 \times 10^{6}$ & $2.1 \times 10^{7}$ \\
\hline \hline
\end{tabular}




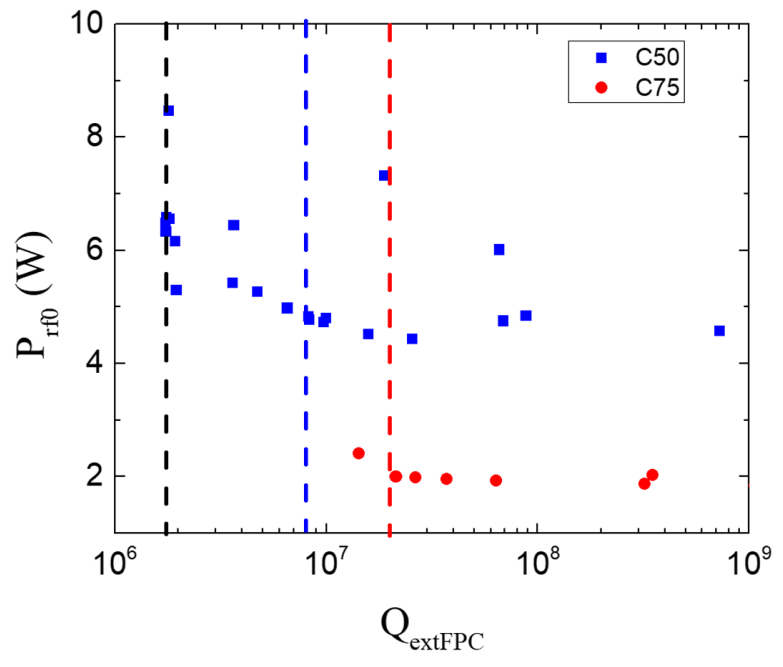

FIG. 19. Computed rf heat load $P_{r f 0}\left(\phi=0^{\circ}\right.$ in eigenmode computations) assuming $T=2.07 \mathrm{~K}$ and $E_{\text {acc }}=7 \mathrm{MV} / \mathrm{m}$ as a function of $Q_{\text {extFPC }}$ for C50 and C75 cavities in the CM configuration. The vertical dashed lines correspond to the design value of $Q_{\text {ext FPC }}$ for a C50 cavity $\left(1.74 \times 10^{6}\right.$ with flush tuner plungers), its specified value of $8.0 \times 10^{6}$ (after bench tuning), and the $Q_{\text {ext FPC }}$ of $2.0 \times 10^{7}$ for a C75 cavity, which is both its specified and design value.

substantial standing waves in the FPC transmission line, the plunger positions have been varied in subsequent computations for both the C50 and C75 CM configurations. Figure 19 plots $P_{r f 0}\left(Q_{\text {ext FPC }}\right)$ obtained from these simulations for direct comparison with the experimental data plotted in Fig. 16. A lesser number of cases has been studied for the C75 CM configuration than for the C50 configuration, since the rf losses are significantly smaller and depend less on stub tuner settings.

The results from the numerical simulations lead to the following observations. (i) The simulation results are in good agreement with experimental data for both the C50 and $\mathrm{C} 75 \mathrm{CM}$ configurations. (ii) The results confirm that the $Q_{0}$ degradation is significantly less in the $\mathrm{C} 75$ cavities than in $\mathrm{C} 50$ cavities due to the cold window losses. The $Q_{0}$ degradation due to the cold window metallization alone covers 57-86\% for the C50 cavities compared to only 9$30 \%$ for the $\mathrm{C} 75$ cavities for the numerical cases studied, depending on the stub tuner settings. (iii) It is difficult to decrease the $Q_{\text {extFPC }}$ below the cavity design value by inserting the plungers. For C50 cavities, the design value refers to the $Q_{\text {extFPC }}$ of as-built cavities, as used in the simulations and not as tuned on the bench, so with an unknown FPC body deformation. A decrease of the $Q_{\text {ext FPC }}$ values below the design values required all plungers to penetrate about halfway into the waveguide, although this does not exclude that other settings exist for lowering the $Q_{\text {extFPC }}$. (iv) $P_{r f}$ values tend to increase on average with decreasing $Q_{\text {extFPC }}$ values, particularly when $Q_{\text {ext FPC }}$ is below the specified value of $8 \times 10^{6}$ for the C50 CM configuration. The actual rf heat load at $2.07 \mathrm{~K}$ can yet depend significantly on the specific stub tuner settings and, thus, the resulting standing-wave amplitude at the cold window position. (v) $P_{r f 0}$ may vary for similar $Q_{\text {ext FPC }}$ values depending on the specific stub tuner settings, which is more pronounced for C50 cavities and for which strong variations occurred within a wide $Q_{\text {ext FPC }}$ range. (vi) A simulation with largely retracted tuner plungers for the C50 configuration reveals that $Q$ results do not differ significantly from those with flush tuner plungers.

Plunger settings have been found that yield a $Q_{\text {ext FPC }}$ value close to $8 \times 10^{6}$ with $P_{r f 0}$ being $\sim 4.8 \mathrm{~W}$ for C50 cavities. While this is realistic in comparison to Fig. 16, one should recall that the CEBAF cavities undergo a plastic bench tuning of the FPC waveguide body to elevate the $Q_{\text {ext FPC }}$ value to the specified value per Table I before CM installation. Thus, the tuned cavities should theoretically yield the specified $Q_{\text {ext FPC }}$ values if the entire transmission line would have a voltage standing-wave ratio of 1 $\left(S_{11}=0\right)$, i.e., being smooth throughout (no rf impedance change), and would exhibit a negligible surface resistance, while the transmission line would be terminated in a matched load. This also implies rather flush plunger positions $(0,0,0)$ in reality. As already discussed in Sec. IVA, the rf model cannot account for the unknown waveguide deformations. Consequently, the plungers have to penetrate into the waveguide to elevate the $Q_{\text {ext FPC }}$ to $8 \times 10^{6}$. This can yield a different standing wave than in reality to obtain the same $Q_{\text {ext FPC}}$, which consequently can explain all or a portion of the difference between measured and simulated rf heat losses.

Overall, the simulation results have delivered rf heat load values comparable to those measured for C50 and C75 cavities in CM 1L13 at the same stored energy in the cavities. This is a very encouraging finding and at the same time explains the magnitude of the $Q_{0}$ degradation of C50 and $\mathrm{C} 75$ cavities observed in CEBAF.

Eventually, the rf losses in the copper-plated stainless steel waveguide section of the FPC between the warm and cold windows shall be assessed for one case resembling the C75 CM configuration with flush plunger settings. The residual resistivity ratio $R R R$ of the copper plating determines the thermal and electrical conductivity. We assume an $R R R$ not better than 30 for the plated copper on stainless steel. The electrical resistivity values at $R R R=30$ have been taken from Ref. [19] given in a temperature range of 1-300 K. Hereby, the resistivity values are rather constant within 1-20 K.

The conceived rf model is depicted in Fig. 20. It incorporates 11 waveguide sections to allow the assignment of sectionwise temperature-dependent conductivity values along the copper-plated waveguide. These sections are differentiated by color ranging from $2.07 \mathrm{~K}$ to ambient temperature. A reasonable equilibrium temperature profile was assumed from 2.07 to $300 \mathrm{~K}$ for a realistic rf heat load 


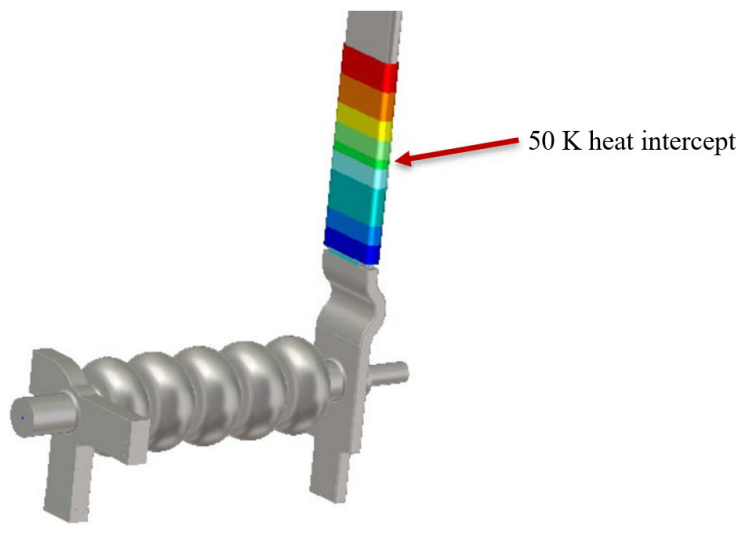

FIG. 20. The rf model for resembling a C75 cavity in a CM configuration including the losses in the copper-plated stainless steel. The copper plating covers the region above the cold window to the warm window (not included in the model). The electrical conductivity of the copper plating with $R R R=30$ ranges from $1.94 \times 10^{9} \mathrm{~S} / \mathrm{m}$ (blue) to $5.7 \times 10^{7} \mathrm{~S} / \mathrm{m}$ (red).

estimate. It was also assumed conservatively that the rf heat conducted into the LHe arises from all the waveguide sections below the $50 \mathrm{~K}$ intercept, including the section at $50 \mathrm{~K}$. This results in a combined $Q$ of $2.8 \times 10^{11}$. The copper losses will therefore degrade the combined $Q_{0}$ by only $\sim 4 \%$.

\section{CONCLUSIONS}

The numerical simulations and experimental data for cavities and doglegs tested in the VTA provide evidence for significant extrinsic of losses related to the metallization of the cold rf window installed on cavities in original CEBAF cryomodules. These extrinsic rf losses are responsible for an $20 \%$ increase in the rf heat load at $2.07 \mathrm{~K}$, even at the low forward power levels required for the VTA tests. The numerical simulations and experimental data for cavities installed in the cryomodules in the CEBAF tunnel indicate that such extrinsic losses can increase the rf heat load at $2.07 \mathrm{~K}$ by an even more significant factor, which yet depends on the stub tuner settings. These results, combined with the additional rf losses in the cavity walls due to the remanent magnetic field in the original CEBAF cryomodules, finally provide an explanation for the large $Q_{0}$ degradation (on the order of $\sim 50 \%$ ) in original CEBAF cryomodules compared to VTA test results since their commissioning in 1991. The presented data thus solve a 30year-old puzzle.

The more recent $\mathrm{C} 75$ cavities have the advantage of requiring a higher $Q_{\text {ext FPC }}$ value by design compared to C20 and C50 cavities. This reduces the external power losses at any given cavity stored energy and makes the C75 cavities much less sensitive to rf losses arising in the cold windows and as a function of the stub tuner settings, as verified experimentally and numerically.
In general, we recommend to not implement cold windows in potential future cavity cryomodule designs to avoid corresponding rf heat losses to occur in the liquid helium but rather place rf windows outside the cavity helium vessel as done for $\mathrm{C} 100$ cavity cryomodules. Future work will pursue the reduction of the MoMn metallized area around the cold window, without compromising the mechanical strength and the UHV leak tightness of the brazed metal to ceramic joint. In parallel, one might conduct $\mathrm{R} \& \mathrm{D}$ aiming at replacing the current MoMn metallization with a less lossy or superconducting alloy, though this might not lead to an immediate breakthrough, since a similarly strong bond of the metallization to the ceramic must be achieved.

\section{ACKNOWLEDGMENTS}

We acknowledge H. Wang for suggesting the experimental procedure used in the measurements of FEL04, M. Drury, F. Humphrey, D. Gelhaar, C. Kerns, L. King, and A. Solopova for helping with the measurements in the FEL vault and the CEBAF tunnel and J. Benesch for the layout in Fig. 4. We thank M. Drury and C. Reece for providing some of the data shown in Fig. 1. Finally, we also thank JLab's SRF cavity production group for carrying out the surface processing, assembly, and some of the cavity rf testing in the VTA. This manuscript has been authored by Jefferson Science Associates, LLC under U.S. Department of Energy Contract No. DE-AC05-06OR23177.

[1] C. W. Leemann, D. R. Douglas, and G. A. Krafft, The continuous electron beam accelerator facility: CEBAF at the Jefferson Laboratory, Annu. Rev. Nucl. Part. Sci. 51, 413 (2001).

[2] C. E. Reece, Continuous wave superconducting radio frequency electron linac for nuclear physics research, Phys. Rev. Accel. Beams 19, 124801 (2016).

[3] M. A. Drury et al., Summary report for the C50 cryomodule project, in Proceedings of the 24th Particle Accelerator Conference, PAC-2011, New York, 2011 (IEEE, New York, 2011), pp. 1044-1046.

[4] M. A. Drury et al., CEBAF upgrade: Cryomodule performance and lessons learned, in Proceedings of the 16th International Conference on RF Superconductivity (SRF'13) (JACoW, Geneva, 2013), pp. 836-843.

[5] R. A. Rimmer et al., Upgraded cavities for the CEBAF cryomodule rework program, in Proceedings of the 18th International Conference on RF Superconductivity (SRF'17) (JACoW, Geneva, 2017), pp. 168-172, https:// doi.org/10.18429/JACoW-SRF2017-MOPB049.

[6] G. Ciovati, G. Cheng, M. Drury, J. Fischer, and R. Geng, Impact of remanent magnetic field on the heat load of original CEBAF cryomodule, IEEE Trans. Appl. Supercond. 27, 1 (2017).

[7] M. A. Drury et al., Commissioning of the prototype C75 cavities in a CEBAF cryomodule, in Proceedings of the 9th 
International Particle Accelerator Conference (IPAC'18) (JACoW, Geneva, 2018), pp. 3961-3964, https://doi.org/ 10.18429/JACoW-IPAC2018-THPAL134.

[8] E. Daly et al., Improved prototype cryomodule for the CEBAF $12 \mathrm{GeV}$ upgrade, in Proceedings of the 20th Particle Accelerator Conference, PAC-2003, Portland, OR, 2003 (IEEE, New York, 2003).

[9] C. Leemann, CEBAF design overview and project status, in Proceedings of the 3rd International Conference on RF Superconductivity (SRF'87) (JACoW, Geneva, 1987).

[10] L. Phillips, C. Reece, T. Powers, and V. Nguyen-Tuong, Some operational characteristics of CEBAF rf windows at $2 \mathrm{~K}$, in Proceedings of the 15th Particle Accelerator Conference, PAC-1993, Washington, DC, 1993 (IEEE, New York, 1993), Vol. 2, pp. 1007-1009.

[11] F. Marhauser, G. Ciovati, G. Cheng, E. F. Daly, M. Drury, A. Freyberger, G. Krafft, T. Powers, and R. Rimmer, C75 cavity specifications and commissioning of the prototype cavity pair, Technical Note No. JLabTN17055, Jefferson Lab, 2017.

[12] S. J. Zinkle and R. H. Goulding, Loss tangent measurements on unirradiated alumina, Technical Note No. DOE/ ER-0313/19, Oak Ridge National Lab, 1996.

[13] N. Alford, J. Breeze, X. Wang, S. Penn, S. Dalla, S. Webb, N. Ljepojevic, and X. Aupi, Dielectric loss of oxide single crystals and polycrystalline analogues from 10 to $320 \mathrm{~K}$, J. Eur. Ceram. Soc. 21, 2605 (2001).

[14] J. Krupka, K. Derzakowski, B. Riddle, and J. Baker-Jarvis, A dielectric resonator for measurements of complex permittivity of low loss dielectric materials as a function of temperature, Meas. Sci. Technol. 9, 1751 (1998).

[15] D. Systemes, CST Studio Suite electromagnetic field simulation software, https://www.3ds.com/productsservices/simulia/products/cst-studio-suite/.

[16] G. Ciovati et al., Cavity production and testing of the first C75 cryomodule for CEBAF, in Proceedings of the 2021 International Conference on RF Superconductivity (SRF'21) (JACoW, Geneva, 2021).

[17] M. Drury, T. Lee, J. Marshall, J. Preble, Q. Saulter, W. Schneider, M. Spata, and M. Wiseman, Commissioning of the CEBAF cryomodules, in Proceedings of the 15th Particle Accelerator Conference, PAC-1993, Washington, DC, 1993 (IEEE, New York, 1993), pp. 841-844.

[18] D. Walzer (private communication).

[19] J. E. Jensen, W. A. Tuttle, R. B. Stewart, H. Brechna, and A. G. Prodell, Brookhaven National Laboratory selected cryogenic handbook, Technical Report No. BNL12000R, Brookhaven National Laboratory, 1980, Vol. II, Secs. XXVIII. 